\title{
Sacrificing America \\ On The Altar Of Mediocrity
}

Les Nunn, University of Southern Indiana, USA

\author{
The Dumbing Down Of America
}

\section{TYPES OF LEARNING}

\author{
William Glasser said we learn: \\ $10 \%$ of what we Read \\ $20 \%$ of what we Hear \\ $30 \%$ of what we See \\ $50 \%$ of what we See and Hear \\ $70 \%$ of what we Discuss with Others \\ $80 \%$ of what we Experience Personally \\ 95\% of what we Teach to Someone Else.
}

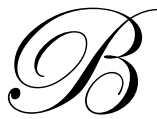

efore going into how we learn, let us look at the types of learning we are involved in as humans. Learning can be broken down into two main categories: associative learning and non-associative learning. (1) (Wood, D.C. (1988). Habituation in Stentor Produced by Mechanoreceptor Channel Modification, Journal of Neuroscience, 2254(8), Mayer, R.E. (2001), Multimedia Learning. New York: Cambridge University Press. ISBN 0-52178-749-1)

Associative learning involves operant conditioning (using consequences to change the occurrence and form of behavior), classical conditioning (the idea of a classical or Pavlovian conditioned response), imprinting (rapid learning at a particular age or life stage that is not related to consequences of behavior), observational learning (the most basic form of learning by watching others), play (activity for the "fun of it" that improves performance in future similar situations), multimedia learning (as kids do with video games), and electronic learning (meaning computer-enhanced learning).

Non-associative learning is by habituation (reactions lessen over time as the individual becomes used to an otherwise scary situation) and sensitization (the response increases following repeated administration of a stimulus).

We can learn by rote (memorization), informally (simply by day-to-day informal situations), and formally (as in a school classroom environment).

Before going into how we learn in the general sense, we must first understand the brain and the physiology of how it functions in the learning process.

\section{HOW THE BRAIN WORKS}

We think with our brain, of course. The brain can be likened to the most sophisticated computer in existence. It controls our every thought and action. It controls the conscious and the unconscious body. Being an organ of the body, it affects and is affected by the body. Like the rest of the body, it grows, becomes stronger, weakens, and dies. 
With this in mind (no pun intended), we wonder how does the brain work? Understanding this can help us know how we think. Understanding how we think can help us know how we learn. Understanding how we learn can help us know how to teach.

\section{Neurons:}

Neurons are the highways of nerve messages traveling from the brain to all parts of the body and back again. Neurons are the nerve cells that transmit signals to and from the brain at speeds up to 200 miles per hour (about the same as traveling the distance of a football field in one second). The human brain has approximately 100 billion neurons. The smallest is 4 microns wide and the largest is 10 microns wide. One micron is one thousandth of a millimeter! (1) (Note: much of the information in this chapter has come from the following sources: nia.nih.gov/Alzheimers/Publications/Unraveling The Mystery; utexas.edu/research/asrec/neuron.html the Neuron, Dr. C. George Boeree, webspace.ship.edu.cgboer/theneuron.html; lpmpjogja.kiknas.go.id/kc/b/brain; www.howourbrainswork.com; $\quad$ Scholarpedia.org/article/Neuron; $\quad$ Wikipedia.org/wiki/neuron; Wikipedia.org/wiki/dendrite; tbiguide.com/howbrainworks.html; en.Wikipedia.org/wiki/limbic_system.)

These neurons are cells composed of long arms (axons) with tiny branches at the end (dendrites). Axons take information away from the cell body and branch further from the cell body. Dendrites, on the other hand bring information to the cell body and branch near the cell body. Axons have been described as the long extension of a nerve cell, and bundles of axons are generally called nerves.

Neurons are the oldest cells in the body and the longest. Some neurons are as much as three feet long. Giraffes, however, have neurons ten to twelve feet long. Many neurons are never replaced when they die, but the hippocampus part of the brain can grow new neurons in adult humans.

At the end of axons are terminals which transmit a signal across a synapse, or gap, between the axon and the receiving nerve cell. A typical neuron has from 1,000 to 10,000 synapses, meaning that each neuron can communicate with as many as 10,000 other neurons, muscle cells, glands and other parts of the body. Remember, there are as many as 100 billion neurons in the adult human brain.

There are different types of neurons: sensory neurons which carry messages from the body's sense receptors (touch, odor, taste, vision, sound) to the central nervous system and are about 1 percent of all neurons; motoneurons carry signals from the central nervous system to muscles and glands; and interneurons neurons constitute the nerve wiring within the central nervous system.

Neuron cells do not divide and grow in the normal sense of cellular growth. Instead, they are permanently amitiotic and do not divide. They do, however, undergo adult neurogenesis. They communicate with each other across chemical and electrical synapses, and can actually change electrical signals into chemical signals to be transmitted on to another neuron.

\section{Glial Cells:}

Approximately ninety percent of the brain cells are glial cells. They are nerve cells, but do not carry any nerve impulses. Instead, they are support neurons. Among their vital functions are to make myelin or the insulation around neurons.

These cells produce chemicals which regulate neurons, store brain chemicals, release certain substances at synapses, and take up and release calcium. We are learning that glial stem cells can be turned into neurons. Recent studies are indicating that people suffering from depression contain fewer glial cells. And, we have found that glial cells produce neurotropic growth factors that may be involved in repairing and re-growing neurons. 


\section{Dendrites:}

Dendrites are the parts of neurons that are extensions of the main body of the neuron and have many, many branches. In fact, neurons can have thousands of branches of dendrites. Dendrites do not secrete any neurotransmitter chemicals and axons do not have chemical receptors. It is a symbiotic relationship between these chemicals and axons. The most important chemicals associated with this are sodium and calcium.

\section{Synapses:}

As indicated, neurons communicate with each other through their synapses or gaps. This is where the axon of once neuron cell comes into contact with another neuron's dendrite. The number of synapses (or connections between brain cells) is astronomical. For instance, each of the brain's 100 billion neurons has an average of 7,000 synaptic connections. This means that the brain of a three-year-old child can have as many as one quadrillion synapses. As we shall see, the number of neurons declines with age, so an adult human could have as many as onehalf a quadrillion synapses.

\section{Electrical Activity of Neurons:}

Messages travel from neuron to neuron through electrical activity. This is accomplished by the flow of positive electrically charged ions, and caused by stimuli, such as pressure, chemical transmitters, different electrical current voltages traveling through the neurons, etc. These nerve impulses do not just happen in varying degrees of intensity. They either happen all the way or not at all. In other words, a signal is either sent completely, or it is not sent at all. Generally, the impulse goes only from the axon to the dendrite.

Neurological diseases, such as Alzheimer's disease, Parkinson's disease, myasthenia gravis, etc. are types of diseases where the transmission of signals through neurons is interrupted or prevented all together. The myelin sheath surrounding the neurons, acting as insulation, can become damaged thereby adversely affecting the ability of the message to travel through the neurons.

\section{Neurotransmitters:}

Neurotransmitters are messages that are produced by the body instructing its different parts, glands, etc. to do something or not to do something. Neurotransmitters essentially affect the entirety of the body. They help control body temperature, blood pressure, insulin levels, and on and on. Each transmitter serves a specific function in controlling the body.

They tell a particular part of the body to do something (excitatory messages) or to not do something (inhibitory messages). For instance, acetylcholine is an excitatory message in charge of learning and memory, while GABA is an inhibitory message controlling thinking, understanding, language and perception. Dopamine, an excitatory message, impacts the pleasure/reward processes of the brain.

\section{Development of the Brain:}

The development of the brain is very interesting. Initially, axons can travel about the developing brain to reach their connective targets. The targets (recognition molecules) can attract or repel axons. Usually, the growing axons do reach the correct target without any problem. However, at times, they do not, resulting in mental retardation, attention disorder, and other maladies.

As the brain develops, this axonal growth continues, even to a degree in adulthood, but not at the same speed. A fetus has many times more neurons before birth than it does after. It is estimated that some ninety percent of the neurons are destroyed during this developmental stage (sometimes at the rate of 200,000 per day) so that at birth only ten percent, or 100 billion, are left. This loss continues, as any parent of a teenager can tell you, until another thirty to fifty percent of the brain's synaptic connections are destroyed during adolescence. 
Even with all of this wonderful knowledge, the workings of the brain are still a mystery. We do not fully understand how long term memory works and many other things. But, we are learning.

\section{The Brain as a Computer:}

The neurons of the brain operate together to be a huge and complex parallel information processing system. When you think if it, the tasks that a brain handles are many, varied, and extremely complex. The brain can learn from experience, heal itself to some degree, controls the functions of the body, handle our self-awareness, and, although it is not totally understood how, the brain gives us our intelligence.

The brain can process 100 trillion instructions per second, where as the most powerful computer in the world can handle 478 trillion instructions per second. However, many other types of comparisons fall by the wayside when considered.

\section{The Parts of the Brain:}

The surface area of the average adult brain is about 450 square inches, which equates to about two pages of a newspaper. Obviously, for this much surface to fit into a person's skull, the brain surface must be folded over itself in most, if not all of its surface. That is, in fact, the case.

\section{Forebrain:}

This part of the brain is divided into the thalamus, hypothalamus and the limbic system. The thalamus is like a relay station for all sensory information. The Hypothalamus regulates basic needs, fighting, fleeing, feeding and mating.

The front of the brain (the forebrain) is the cerebral cortex controlling the higher functions and the mid and hindbrain taking care of the autonomic, unconscious functions. The neocortex, accounting for 75 percent of the brain mass, handles language and consciousness. It is interesting to note that the neocortex of the human brain is much larger than the neocortexes of other animals. Since this part of the brain deals with language and consciousness, it might explain why humans have the most developed language and complicated methods of communication of all animals.

Neurons have been compared with microchips. It would be difficult for computer software writers to program the brain because it operates parallel and interconnected. While it is "hard-wired" to handle certain functions (ability to learn language, for instance, and instincts), it is rapidly able to adapt, learn from experience, and deal with emotions, as well as handle other activities requiring instant interrelated thought processing. Very few, if any, computers could handle this.

\section{Brainstem:}

The brainstem is composed of three parts: medulla, pons and cerebellum. The medulla controls breathing, circulation, and muscle tone. The pons connects the brainstem with the cerebellum and helps to control sleep and arousal. The cerebellum takes care of coordination of voluntary movement and equilibrium.

\section{Midbrain:}

The midbrain becomes involved in sensory processes and dopamine production. At the same time, it affects muscle reflexes and breathing.

\section{Cerebral Cortex:}

This is where the most important conscious activities are controlled, such as learning, memory, thinking, consciousness, etc. 


\section{Hemispheres:}

The left hemisphere of the brain is involved in systematic, logical interpretation of information and symbols, mathematics, abstraction, reasoning, etc. The right hemisphere processes multi-sensory input to provide a "holistic" view of a person's environment. Visual, spatial skills, dancing, gymnastics, are all processed here.

\section{Frontal Lobe:}

This part of the brain deals with the ability to concentrate, judgment, personality and emotional traits.

\section{Limbic System:}

The limbic system includes the cerebral cortex, hippocampus, hypothalamus, thalamus, and other structures of the brain. The hippocampus which is key to learning and memory. It is also associated with controlling of emotions such as sex, anger, fear, etc, and motivation, recent motivation and biological rhythms.

Basically, it affects the endocrine system and the automatic nervous system. Connected to the brain's pleasure center, it plays a role in sexual arousal and "highs" received from certain drugs. Also, a strong flow of information to the prefrontal cortex helps to bring a pleasurable feeling when solving problems. This connection can be surgically severed to treat or cure some emotional disorders.

Unfortunately, the limbic system is tremendously impacted by use and abuse of substances like alcohol, nicotine, and other drugs.

\section{Functions of the Brain:}

The brain regulates our respiration, heartbeat, blood pressure, endocrine system, body movement, body temperature, ... the list could go on and on. Recent developments in neuroimaging have allowed scientists to learn what parts of the brain control what activities. When making decisions with moral implications, two areas of the brain show increased activity - the area involving emotions and that involved with abstract reasoning. This illustrates that the brain is capable of considering both emotional and logical issues regarding the same problem.

Experiments by Dr. Kevin Dunbar of Dartmouth College have indicated that when scientists perform experiments and the results are different from what had been anticipated, the women scientists are more likely to continue experimenting to find out why. Men, on the other hand, probably will ignore the unexpected finding as being something that hinders reaching their goal.

All of the expensive equipment and facilities which educators seem to believe is essential to a good education might not be so necessary after all. Aristotle taught under a tree, without an elaborate building as his school. Likewise, Victoria Rosin, a science teacher in St. Ann's School in Wisconsin does not use textbooks or lab manuals. She teaches by having students create their own experiments and constantly questions their thinking and asks them to explain themselves. She teaches students to ask why things are the way they are. Contrary to public thinking, researchers have found that even sixth graders can deal with complex abstract reasoning.

\section{Cognitive Development of the Brain:}

The brain does not develop all at the same time. Its development in the fetus occurs incrementally and the same is true, to a large part, after birth. The cerebellum is one of the first parts to develop and that controls motor activities. The old saying "use it or lose it" is true for the brain as well as most any other part of the body. It especially applies to development of motor skills in the child.

Times are a'changing, and some of those changes are good and some not so good. As a people, Americans are becoming more and more sedentary at all ages of life. Even in comparing the 1960's to the 1990's we see drastic changes for the worse. In 1960, children spent about 100 hours in a car seat, but in 1995, that had increased 
to 500 hours. Five times longer sitting in just thirty years. Obviously, more sitting means less activity for those children. More sitting means less moving around. Less moving around means less use of motor skills. Less use of motor skills means less development of motor skills.

Statistical data reflects the same increase in sedentary life for children in other activities as well. In most any area you want to compare, you would find the same. Far more time today is spent by children watching television and playing video games and far less time is spent outside running around and playing in physical activities. The physical movement is vital to learning and development of the brain.

Good learning is enhanced by good environment. That is not dependent upon nice buildings. It is dependent upon an enriched environment with mental and physical challenges to learn to overcome. It is an environment where there is less threat and more support and encouragement. Challenge is important, mentally and physically. Learning to overcome difficulties is an essential part of life. This occurs only through encouragement and support from a loving parent and teacher or other person as the child experiences life's challenges.

As demonstrated with the Mozart experiments, a relaxed environment promoting alertness does indeed stimulate, develop and strengthen the brain's connections. While we have learned more about the brain in the past ten years than perhaps over any other period in history, we still have a long way to go in changing our approaches to learning.

\section{Synaptic Mechanisms of Memory:}

Synapses, the connections between neurons, are where messages change and are stored in the brain. While much is known about synapses, there still many mysteries. We know, for instance that the brain ignores more than 90 percent of what it receives. Synapses can change their effectiveness in receiving and transmitting data along the neuron chain. This ability to change is called synaptic plasticity. Some think this is the very basis of learning and of memory itself.

The Brain Sciences Program at Brown University is one of the leaders in studying this synaptic phenomenon. Among the things being analyzed is how "ephemeral episodes of neural activity are transformed into long-lasting changes in synaptic strength.” (__ ( www.brainscience.brown.edu/research/6questions/)

For me, personally, the subject is fascinating. It involves the study of the creation and synthesis of new proteins (many of which come from the translation of mRNAs at the synapses), sequestering the message transmissions at remote locations, triggering their translation in response to synaptic activity, cytoplasmic polyadenylation (which regulates the local translation of messages), and numerous other combinations of biology, neurology, psychology, nutrition, and many other sciences combined. In short, science is just barely scratching the surface of the brain and how it operates. What a glorious time to be alive as we learn more and more about more and more!!!!

\section{Reticular Formation:}

The Reticular Formation (RF) and its Reticular Activating System (RAS) is located in the brain stem and is similar to your little finger in shape and size. It contains millions of neurons. It is the only part of the brain that has access to all incoming information, which it immediately scans and prioritizes, selecting proper responses and then communicates with the rest of the body. And almost nothing is known about this most important part of the brain.

It has been described by Eugene B. Shea in his very informative paper as the "physical basis of consciousness, the brain's chief watchguard." (_) (Shea, E.B., How the Brain Works, www.howourbrainswork.com) Dr. Shea goes on to say that it determines which of the many bits of information are important enough - or novel enough - to report to the higher portions of the brain. The RF can, by itself, handle such matters as digestion, heartbeat, respiration, and the like. 
Think of all that your eyes take in during a day. Yet, your attention is called only to those things your brain (the RF) knows are important to you or are really unusual and may require conscious analysis. Add to that the constant millions of bits of information that come to your brain every second as to blood pressure, blood sugar levels, respiration rates, body temperature, and on and on. The RF, then, filters out over 99 percent of the impulses your brain receives.

As Dr. Shea says "From all the evidence, the RF/RAS can only be characterized as a computer/servoorganism which receives all incoming data, scans and prioritizes that data for further processing in accordance with its programs, and, through the Reticular Activating system which uses the entire brain, generates and controls responses or response impulses "appropriate" to its iterations of the data." (__ ( ( ) (ibid) It brings to consciousness those things which require conscious thought and handles by itself all other matters.

\section{Brain Trauma:}

The technical name for injury to the brain is contrecoup, resulting from the brain being slammed against the skull. Normally, in this kind of injury, the patient suffers diffuse axonal injury or axonal shearing. The long axons are severed in the violent banging of the brain against the skull, so that the nerve is not able to transmit signals any more.

When the axon is broken open, calcium enters into the cell through the open cut and causes the axon to swell. Chemicals that break down proteins (called proteases) are then activated in the axon. Technically, the calcium activates caspases and other enzymes which damage the cell's DNA and bring about a programmed death to the neuron cell.

While it is true in large part that the brain cannot generate new cells, we see the brain try to repair itself. The milder the banging of the brain against the skull, the more likely successful repairing (at least to some degree) can occur. Neurons can grow more fibers which expand to fill in the void created by the deceased axon. Surprisingly, these fibers can develop into dendrites so that neural communications can resume to some degree with neighboring neurons. The re-growth can be hampered or stopped completely by neuroexcitation, hypoxia, hypotension, etc.

Recovery from brain trauma is a complicated topic, and is not addressed any further in this paper.

\section{NUTRITION AND THE BRAIN}

The brain is the hungriest of all the body's organs. Brain cells require twice as much food as other cells in the body. But, they require the right kinds of food to function properly. It goes without saying, but junk food is terrible for the brain, just as they are terrible for the rest of your body. Generally speaking,

- $\quad$ Omega-3 fatty acids build up your brain.

- $\quad$ Proteins tie the brain cells together so they can function well.

- $\quad$ Carbohydrates fuel the brain

- Micronutrients protect the brain.

\section{Omega-3 Fatty Acids:}

Omega-3 fatty acids are necessary for the brain. They form the outer membrane of the cells of the brain and are involved in learning and memory connections between neurons. New membranes are formed to protect these neurons, and Omega-3 is the best for good brain functions. Omega-3 comes primarily from fish.

Choline, found in B vitamins, enhances memory. Other good foods would be avocados, fresh coconut, and extra virgin olive oil. Avoid all trans fats! 


\section{Proteins:}

Essential amino acids are needed for neurotransmitter production. They come from the breaking down of proteins in the digestive process. So, proteins are vitally necessary for good brain functioning. Proteins from meats, eggs, milk products, dried beans, green leafy vegetables, nuts and seeds are all good.

\section{Carbohydrates:}

Carbohydrates fuel the brain. The brain needs proper levels of glucose, which comes from carbohydrates. But, it is essential that you avoid blood sugar spikes.

The brain monitors blood sugar levels. Starchy carbohydrates are digested quickly and turned into sugar which is absorbed by the blood stream. Too much starchy carbohydrates by themselves cause a spike up in the blood sugar levels. The brain then tells the pancreas to produce more insulin which is then dumped into the blood to lower the blood sugar level. This up and down is not good for the brain or the body. Starchy carbohydrates include such foods as corn, potatoes, carrots, beets and the like. If you are going to eat starchy carbs, combine them with protein, which slows down the digestion process, thereby giving a much slower input of sugar into the blood stream. The repeated high inputs of sugar into the blood causing the excessive production and dumping by the pancreas of insulin into the blood stream is the leading cause of diabetes.

\section{Micronutrients:}

Micronutrients, such as minerals, are found naturally in the fruits and vegetables we eat. Most nutritionists recommend at least five helpings of fruits and vegetables per day and ten per day to help prevent cancer. Also, minerals and vitamins are necessary for the digestion process to convert amino acids into neurotransmitters.

Key neurotransmitters include:

Acetylcholine (ACh) which excites other neurons and is involved in memory, voluntary movement of muscles, and the like. Low ACh levels are associated with Alzheimer's disease.

Dopamine which is involved in muscle movement, attention span, learning, and emotional arousal. Low dopamine levels are found in Parkinson's disease patients and high dopamine levels are found in people with schizophrenia. Dopamine is found in all protein.

Serotonin which affects arousal, sleep, mood changes, appetite, sensitivity, feelings of pleasure, etc. Prozac extends serotonin activity. Good serotonin foods include starchy vegetables, potatoes, pasta, cereals, breads, etc.

\section{Good Brain Meals:}

Good meals for the brain would include as follows:

\section{Breakfast:}

Avoid caffeine. Caffeine might increase mental capacity for a little while, but when it is over, there is a corresponding drop in energy. The only way to keep it at a high level is to drink more and more caffeine as the day goes on. This is harmful to the body.

Avoid sugar. As explained above, sugar is a simple carbohydrate that is digested quickly and absorbed by the blood. This triggers the pancreas dumping insulin into the blood stream to lower the high sugar level, which can lead to diabetes. 
Long chain carbohydrates are good as they take longer to digest, are absorbed gradually by the blood stream and provide glucose. Glucose is the fuel for the brain and mental exercise drains the brain of its glucose supply. to neuron.

Egg yolk supplies choline which boosts brainpower in that it speeds up the sending of signals from neuron

Lunch:

Make the noon meal your dinner. This is the way it was 100 years ago when people were involved in so much physical labor. They needed a big breakfast to get them through the morning and a big noon meal to get them through the afternoon. Then came a light supper. Eat a noon meal of the proteins and other foods described above.

Supper:

A light supper will help you sleep better and deeper. You do not need a lot of calories at night. They simply turn to fat because your body is not physically active after you eat supper and the extra nutrition is stored as fat.

Drinks:

It is very important to drink enough water and fluids. The brain needs adequate fluids to function properly. Some recommend 80 ounces of water a day. Avoid caffeine! Avoid soft drinks. Soft drinks contain much sugar, which causes the insulin problems described above.

\section{Food as a Stimulus:}

Studies by the United States Department of Energy's Brookhaven National Laboratory in Upton, NY have shown that the brain circuits involved in addiction are activated by the sight of food, odor of food, and even the thought of food. Americans are constantly being bombarded by food images on television, news print, billboards, etc. All of this activates the right orbito-frontal cortex of the brain, which is the same part of the brain involved when addicted people crave drugs. It increases the levels of dopamine (involved in pleasure and reward). The same parts of the brain are excited in obese people as in drug addicts when they see food.

In fact, food stimulations increase all activity in the brain. Metabolism was higher in all parts of the brain. Simply seeing food pictures will increase a person's feeling of hunger, even though their body does not heed food. (__ (Brookhaven national Laboratory News, April 19, 2004) 
NOTES 